Copyright Philosophy of Science 2014 Preprint (not copyedited or formatted)

Please use DOI when citing or quoting

Forthcoming in: Philosophy of Science

\title{
Experimental Modeling in Biology: In Vivo Representation and Stand-ins As Modeling Strategies
}

\author{
Marcel Weber \\ Department of Philosophy \\ University of Geneva \\ marcel.weber@unige.ch
}

\begin{abstract}
Experimental modeling in biology involves the use of living organisms (not necessarily socalled "model organisms") in order to model or simulate biological processes. I argue here that experimental modeling is a bona fide form of scientific modeling that plays an epistemic role that is distinct from that of ordinary biological experiments. What distinguishes them from ordinary experiments is that they use what I call "in vivo representations" where one kind of causal process is used to stand in for a physically different kind of process. I discuss the advantages of this approach in the context of evolutionary biology.
\end{abstract}

\section{Acknowledgements}

I wish to thank Kristina Hillesland, Mike Travisano, Ken Waters and the San Diego audience for an exciting PSA 2012 symposium on experimental modeling. This paper also benefited from discussion with audiences at the LMU Munich, the Sorbonne and the Second European Advanced Seminar in the Philosophy of the Life Sciences (Hermance). 
Copyright Philosophy of Science 2014 Preprint (not copyedited or formatted)

Please use DOI when citing or quoting

\section{Introduction}

A growing body of scholarship shows that experiments play a variety of different roles in the biological sciences (see Weber 2012 for an overview). In addition to the epistemic roles traditionally associated with them - hypothesis testing and supporting causal inferences experiments can sometimes also play exploratory or preparatory roles (e.g., Burian 1997; Weber 2005; O'Malley 2007; Elliott 2007). Furthermore, new experimental approaches can have a dramatic impact on the practice of biology; they have even been shown to steer biological research into entirely new directions without the help of any new theories (Rheinberger 1997). In this paper, I want to examine the epistemic role of a kind of experimentation that is becoming more important in biological research but that has not been much discussed in the literature: experimental modeling.

To a first approximation, I mean by "experimental modeling" the construction and study of a specific kind of model system, namely a model system that is composed of living organisms, not necessarily "model organisms" in the sense in which biologists use that term. Model organisms in the latter sense play a variety of different roles in biological research (Weber 2005; Leonelli and Ankeny 2011; Love and Travisano 2013). Here, I am interested in a very specific epistemic use of living organisms, namely in order to re-create or simulate specific biological processes. These organisms need not be model organisms in the usual sense, even though they often are. I will examine the epistemic role of such experimental models and contrast it both with other forms of experimentation as well as with other forms of modeling. My goal is to show that the epistemic role of experimental models is quite unlike that of ordinary experiments and more akin to the role of theoretical models.

To give some classic examples of what I mean by experimental modeling, evolutionary biologists have been re-creating evolutionary processes in the laboratory by using fruit flies as early as the 1930s (e.g., Timoféeff-Ressovsky 1934). I will present some more recent examples of experimental evolution in Section 3A. In a similar way, $20^{\text {th }}$ Century ecologists have staged interspecies-competitions in artificial communities or microcosms (e.g., Park 1948). Today, as we shall see (Section 3B), experimental ecologists can engineer synthetic ecological systems with very specific properties in order to study ecological and evolutionary processes.

In the following section, I will try to locate experimental models in the conceptual landscape of models, experiments, and simulations. I will show that a crucial difficulty lies in distinguishing the epistemic role of experimental models from that of ordinary experiments. In Section 3, I will introduce two examples of experimental modeling, both from the area of experimental evolution. Section 4 examines to what extent these experimental models provide causal knowledge, as the production of this kind of knowledge is traditionally seen as the primary role of experiments. In Section 5, I further characterize the epistemic role of the 
experimental models by using the notions of stand-in and in vivo representation. Section 6 tries to answer the question of what epistemic advantages this approach offers, in particular as compared to the computational or "in silico" approach. Section 7 summarizes my conclusions.

\section{Models, Experiments, and Simulations}

As a number of authors have pointed out, the construction and study of material, biological systems may sometimes play a similar or even the same epistemic role as theorizing (e.g., Griesemer 1990; Plutynski 2001; Waters 2012). On this view, Park's (1948) experimental work involving flour beetles should be considered to be analogous to Lotka's and and Volterra's well-known mathematical models of interspecific competition; they are both forms of theorizing, but with different means. Park used living organisms while Lotka and Volterra used calculus and differential equations to model competition in ecological communities. Both models are idealized; the conditions they create or assume do not exist in the wild. Also, both approaches are rigorous in their own way: Lotka-Volterra's work was mathematically rigorous while Park's work was rigorous at the level of experimental design and control. Finally, much like mathematical models, experimental models allow scientists to explore the space of what is possible in nature. ${ }^{1}$ Thus, there are many important similarities between experimental models and theoretical models.

Much like experiments, scientific models can also play a variety of epistemic roles (Morgan and Morrison eds. 2005; Frigg and Hartmann 2012). But it seems that a standard role of a theoretical models in science is to selectively represent some aspects of a kind of system or process, which may be real or fictive (e.g., Giere 2004; Frigg 2010; Weisberg 2013). A distinctive feature of models seems to be that they represent their target in an indirect way (Weisberg 2007). When engaging in theoretical modeling, scientists investigate a surrogate or stand-in for the systems they are interested in, because that stand-in is epistemically more tractable. Another feature is that models come in different forms: Some are abstract, consisting of mathematical equations some of the symbols of which are taken to represent physical magnitudes. But there are also concrete models, for example, the Watson-Crick model of the double helix as it was built from cardboard and wire in 1953.

If physical systems can function as theoretical models, then so can living organisms. Indeed, several authors have suggested that so-called "model organisms" such as Drosophila melanogaster, E. coli, or Arabidopsis thaliana, which have been extremely important in biological research during the last 100 years or so, are basically concrete theoretical models (e.g., Leonelli and Ankeny 2011; Frigg and Hartmann 2012; Weisberg 2013). While I disagree

\footnotetext{
${ }^{1}$ This aspect is particularly emphasized by Plutynski (2001) as well as by Waters (2012).
} 
with this view (as do Levy and Currie forthcoming), the case is different for the experimental models under consideration here. ${ }^{2}$

If we accept the claim that experimental models may serve a similar or even the same role as theoretical models, we should be able to say more clearly what distinguishes their epistemic role from that of experiments traditionally conceived. The existing works on experimental modeling cited above do not address this question. But this question is important because if we cannot answer it we are driven towards the conclusion that all experiments are also theoretical models, a claim that strikes me as counterintuitive. ${ }^{3}$ Note also that what is considered to be a distinctive feature of models, the indirect way in which they represent their target, may also be said about certain experiments. For example, ecologists who want to study the effect of increased atmospheric $\mathrm{CO}_{2}$ levels on forests will not experiment on a whole forest but on a suitably fenced part of a forest. ${ }^{4}$ Thus, experiments may also use some sort of a stand-in for their target.

To add to the confusion, at least some experimental models can also be viewed as simulations, a scientific category that also turned out to be difficult to distinguish conceptually from experiments. Recent discussion on this issue has centered on the question of the extent to which the "materiality" or the stuff of which the systems studied and the target systems are made of matters conceptually and epistemically (e.g., Guala 2002; Morgan 2003, 2005; Parker 2009; Winsberg 2009). In our present examples, it is difficult to decide what should count as relevantly the same stuff, but I will argue later that in experimental modeling causal processes may be used to represent physically different processes (see Section 5).

Winsberg (2009) has argued that the pertinent difference between simulations and experiments lies in the way in which their external validity (i.e., their representativeness with respect to the intended target systems) is justified. According to him, the external validity of a simulation is justified with recourse to the reliability of the principles used for constructing the simulation model. This is knowledge that concerns primarily the target. By contrast, the external validity

${ }^{2}$ In a nutshell, my argument against model organisms being theoretical models is that any theoretical model must be associated with a mapping function that specifies what part or aspect of the model is supposed to represent or stand for what (e.g., that the function symbol " $F$ " in a mechanical model stands for the mechanical force). Such a function is not uniquely defined for a biological model organism such as $E$. coli because such organisms may serve a variety of different purposes, only some of which are representational. By contrast, the experimental models under consideration here are associated with such a function - they are used to represent some specific kind of biological process.

${ }^{3}$ Mäki (2005) defends this claim nonetheless.

${ }^{4}$ I owe this example to Bruno Strasser. 
of experiments is ascertained by an appeal to different kinds of knowledge, including knowledge that the objects of the experiment are properly isolated or shielded from disturbing influences and that the object belongs to the same (relevant) kind as the target.

According to Winsberg's distinction, experimental models such as the ones considered here all qualify as experiments, because much knowledge about the experimental objects is needed to argue for external validity. Thus, his proposal - whatever its merits with respect to the epistemology of simulations - does not help to distinguish between experimental models and ordinary experiments.

Before moving on, I should briefly clarify what I mean by an "ordinary" experiment. For the purposes of this paper I suggest the following: A principal role of biological experiments is the discovery of specific causal relations (Weber 2012). In other words, ordinary experiments serve to provide premises for causal inference. To give an example, the main purpose of the legendary "PaJaMo" experiment in molecular biology—named after the three scientists who designed and performed it in 1959, Arthur Pardee, François Jacob and Jacques Monod-was to establish that the induction of transcription in the $E$. coli lac operon is caused by the release of a repressor protein from a specific DNA binding site (see Weber 2005, Ch. 3 for details). I take this example to be paradigmatic for much experimental activity in biological research. What I want to show here is that experimental models play a somewhat different role, indeed a role that is more similar to theoretical models.

\section{Two Examples of Experimental Modeling}

\section{Example A. Experimental Evolution of Multicellularity}

Traditionally, studies in experimental evolution have focused on adaptation by natural selection (e.g., Lenski and Travisano 1994). More recently, it has become possible to model a major evolutionary transition by using a micro-organism, the much-used yeast Saccharomyces cerevisiae. Ratcliff et al. (2012) subjected yeast cells grown in culture to a selection regime that favors rapid settling. To this end, they used gravity to let liquid yeast cultures settle before transferring the cells in the lower region of the culture tube to fresh medium. This selection regime basically selects for larger size. They found that the yeast cells rapidly evolved to form aggregates with a snowflake-like appearance. This phenotype was not a result of cells clumping together, but of post-division adhesion. So, genetically identical cells stay together. What this does in evolutionary terms is that between-cell variability in fitness is strongly reduced within the multicellular aggregates. Thus, the multicellular yeast of Ratcliff et al. satisfies an important evolutionary criterion for biological individuality (Clarke 2012).

This experiment showed that multicellularity can evolve over a few generations when there is a selective environment that favors aggregates of cells over single cells. It should be noted that the rapid settling phenotype was chosen not because this is thought to be an important factor in 
the evolution of multicellularity (even though it might have played a role). This phenotype was simply easy to select in an experimental regime.

The Travisano lab was also able to demonstrate the evolution of something like a germ linesoma differentiation. What they observed was that in each multicellular individual a few cells become destined for apoptosis (programmed cell death). These dying cells can cause parts of the aggregates to break away. This breaking-up can be classified as the equivalent of reproduction because it produces new individuals that go on to live independently (that is, their descendants may differ in fitness from the parent). Thus, the yeast had evolved a mechanism that maintains a germ line-soma distinction; germ line cells being those that break away from the parent organism to start a new individual.

Travisano and his fellow experimental evolutionists conclude that their yeast system provides a model for the first steps of the evolution to multicellularity, including the emergence of the germline-soma separation.

\section{Example B. Synthetic Ecosystems}

In ecology, there is a long tradition of using so-called "microcosms" to study the interactions that occur between different species sharing a habitat. Microcosms are artificially created and maintained assemblages of different organisms, usually (but not necessarily) kept in a closed container. The examples I would like to talk about here are quite simple microcosms; they just consist of two species or two different strains of the same species that are cultured together in some well-defined environment. I will concentrate on experiments done on microcosms composed of yeast or bacteria grown in liquid culture. In such experiments, two different species of microorganism or two different strains of the same species are cultured together and observed over a number of generations. The purpose of such experiments is to study ecological interactions, in particular mutualism (generally known as "symbiosis"; biologists sometimes use this term more broadly for any kind of community life where there is some kind of interaction between species), parasitism, predator-prey relations, as well as resource competition. Of course, it is also possible to observe evolutionary change in such microcosms.

There are different ways in which such model systems can be set up: First, biologists can simply take two strains as they were isolated from the wild or cultured in the laboratory, coculture them together and see what happens on the short term (ecological timescale, i.e., without sufficient time for significant evolutionary change). Second, evolutionary change can be observed as the two strains / species adapt to each other. Third, it is possible to genetically engineer organisms, thus creating a kind of interaction that they would not engage in (or less strongly so) in their unmodified form. The classic competition experiments by Thomas Park using flour beetles (Park 1948) were already mentioned. They exemplify the first kind of experimental ecology model. 
An example of the second kind is found in the work of Kristina Hillesland and David Stahl (2010). They co-cultured the bacterium Desulfovibrio vulgaris and the archaeon Methanococcus maripuladis. D. vulgaris lives by fermenting lactate, thus producing acetate, carbon dioxide and reduced hydrogen (this is somewhat different from the better known forms of fermentation that produce ethanol instead of acetate and hydrogen). This reaction does not provide much energy unless the concentration of the products is kept very low. This is exactly what Methanococcus does; it lunches on carbon dioxide and hydrogen by producing methane. Thus, a mutualistic relationship is established between the two species. Hillesland and Stahl used this system to study the evolution of the mutualism. They showed that the productivity of this system (i.e., the amount of new biomass formed) increased over time due to evolutionary adaptations in both species. However, there were also experimental runs where both species showed instability and eventually extinction. Pairings of evolved strains with the ancestral genotype showed that both species contributed to the increases in productivity, thus both seem to be subject to evolutionary adaptation to the symbiosis with the other strain. An unexpected finding was that the increase in productivity observed was less than the sum of the strains grown in the presence of the ancestral strain. A possible explanation for is that the mutations responsible for the adaptation have some antagonistic pleiotrophic effects.

Examples of the third kind are also prevalent in the literature. For example, Shou, Ram, and Vilar (2007) engineered pairs of yeast strains so that one strain was deficient for the synthesis of an amino acid that the other was able to produce, and vice versa. This introduced a mutualistic interaction between the two strains where there was none before. This system showed some behaviors that were predicted by mathematical models, such as density oscillations in both symbiont populations. In addition, there were some unexpected phenomena. For example, the system showed a tendency to evolve an increased ability to survive rapid drops in population density due to oscillatory dynamics.

To get the analysis of the experimental modeling approach started I would like to draw attention to the similarity of all the cases that I have presented, including the counter-example (PaJaMo). All of them involve intricate experimental manipulations on organisms in vivo. In all of the experiments, a more or less artificial system was produced. However, there are different kinds of artificiality. In the experimental evolution case (example A), no massive manipulations on the experimental organism itself were performed. However, the environmental conditions under which the Travisano lab studied the evolution of multicellularity were quite artificial: only one clone of yeast was present in their cultures. Furthermore, the environmental conditions were kept constant and homogeneous (by a culture regime involving daily transfer and dilution into fresh medium). Such conditions do hardly occur in nature. So here, the artificiality concerned mainly the culture conditions. 
In some of microcosm experiments (example B), the artificiality consisted also in the culture conditions, but additionally in the simplified composition of the ecosystems investigated. Natural communities are much more complex than the two-species or even two straincommunities used in the synthetic ecological communities that I have presented. Furthermore, the biologists genetically engineered their experimental organisms in some of these experiments.

So far, all that has been said about the two examples also pertains to experiments traditionally conceived. I suggest that, in order to see the difference to ordinary experiments, we must examine what kinds of knowledge experimental models help to produce.

\section{What Kind of Knowledge Does Experimental Modeling Provide?}

The evolution of multicellularity experiments (example A) established the causal relevance of gravity in the evolutionary transition from uni- to multicellularity in this system. However, it should be noted that this was not the main purpose of these experiments. The experimental model was designed to study an important evolutionary transition in general, not specifically the effect of gravity on the evolution of yeast cells. Thus, the experiment served not primarily to establish a claim of causal relevance. Rather, the system was already designed such that a certain relation of causal relevance holds, namely a fitness relevance of gravity. To be sure, the experiment did show that rapid settling was a cause of multicellularity in this system. But the main point of the experiment was to show that, if multicellularity is favored by natural selection, mechanisms that realize multicellularity evolve within a few generations. These mechanisms include post-division cell adhesion and the beginnings of soma-germ line distinction. Thus, the experiment probed primarily for the dynamics of an evolutionary transition, not for some specific kind of causal dependence.

The case is somewhat different in example B. There, like in example A, the system was also already designed to exhibit a set of causal dependencies, namely the mutual dependence of the two species on each other as a source of metabolically available energy. It was clear that this mutual dependence would cause evolutionary change. The main question that the experiment sought to answer was how this evolutionary change would occur: Does one species adapt to the other, or do they both undergo adaptive change? Does the interaction always lead to a stable mutualism or is it prone to instability, even to occasional extinction events? Does the stability increase over evolutionary time?

While these experiments also investigated the dynamics of evolutionary change, some of these questions can also be understood as questions about causality: Does the ecological interaction between two species cause adaptations in one or in both species? Does co-evolution cause a state of greater stability over time? Thus, the experimental inquiry had an important causal aspect. But the point of this study was not merely to establish that an evolution towards greater 
stability occurred in this type of ecological interaction. The idea is that the evolutionary changes observed generalize to other kinds of ecological mutualistic interaction between species, interactions that are mediated by physically different kinds of processes. Indeed, quite similar behaviors appear to have been observed by Hillesland and Stahl with their symbiotic methanotrophic bacteria and by Shou et al. and their symbiotic yeast. But the nature of the ecological interaction was quite different: In the first case, it was a symbiosis involving energy-rich foodstuffs, in the second case essential amino acids needed for protein synthesis.

Thus, we have identified an important difference between experimental models and ordinary experiments: While ordinary experiments seek to establish a causal dependence in a particular system, experimental models provide knowledge of causal processes that generalizes to systems where biologically and chemically quite different kinds of causes are at work.

In the following section, I shall compare experimental models to other kinds of scientific models.

\section{Models, Stand-ins, and the Technique of In vivo Representation}

Scientific models come in different forms: some abstract, some concrete, some mathematical, others physical or computational. According to a number of authors, models represent fictive model systems (e.g., Frigg 2010). Typical model systems known from physics are the ideal gas, Bohr's model of the atom or the harmonic oscillator. Physicists invent such models and attribute them with certain properties (e.g., point masses in the case of the ideal gas, frictionless motion in the case of the harmonic oscillator). Then, they study what other properties result from the properties that they built into their fictive model systems, given certain assumptions about how physical properties are related (e.g., Newton's laws). They use mathematical or sometimes computational or simulation methods in order to find out about these properties. Finally, physicists study how similar the model systems are to real physical systems.

I claim that experimental models share important features with such abstract, physical models. They also involve the construction of idealized model systems that do not exist in nature (e.g., an evolving isolated clone of bacteria in a homogeneous environment, an isolated two-species mutualism or an isolated two species-predator prey system, etc). The model is endowed with certain properties that scientists deem important on the grounds of previous theoretical considerations. Then, the scientists study what other properties result from these properties that were built into the model systems. Finally, they compare how similar the model systems are to real systems in nature. Instead of mathematical derivations and computer simulations, they use living organisms. The model systems they study are not fictions, they are real, but no less idealized than classical model systems. 
Another similarity in particular to computational models is the following. Experimental models use certain kinds of interactions to stand in for other biological interactions. For example, in the Travisano lab's experiment on the evolution of multicellularity (Section 3A), the post-division cell adhesion between yeast cells stands in for the adhesive interactions between cells of other multi-cellular organisms. Similarly, the gravity-induced settling stands in for whatever environmental condition favored multicellularity in our evolutionary past. By the same token, in Hillesland and Stahl's work on mutualism as well as in Shou et al. (Section $3 \mathrm{~B}$ ), the metabolic dependence of the two microbial strains used stands in for all kinds of mutualistic interactions between species. ${ }^{5}$

Note that, in all of these cases, the interactions represented by the interaction that stands in for them are multiply realizable by different biological processes. This, I claim, is similar to computational modeling where the physical processes occurring in a computer stand in for whatever interactions are being modeled. Computational algorithms, too, are multiply realizable by physical processes. Thus, experimental models bear many of the marks that are distinctive of theoretical models, marks that are not characteristic of what I call "ordinary" biological experiments.

As I already mentioned in the introduction, it could be objected that all experiments involve some kind of stand-in relation. I agree, but I maintain that we have a different kind of standing-in in experimental models, namely one where the process recreated in the laboratory can represent physically distinct kinds of processes. This is not the case in ordinary biological experiments, where whatever happens in the experiment is physically of the same kind as what happens in the target systems. Consider, for example, the PaJaMo experiment mentioned in Section 2. There, the genetic processes that occurred in the experimental sample were physically identical to the target processes - tokens of lac operon and repressor protein binding to it. ${ }^{6}$

\footnotetext{
${ }^{5}$ It is even possible to model a predator-prey interaction between E. coli strains where one strain kills the other by release of a substance and is at the same time saved by the other strain (Balagaddé et al. 2008). In this experimental model, the release of a toxic substance by the "predator" and of a substance by the "prey" that rescues the predator stands in for the relations of eating and being eaten.

${ }^{6}$ I do not wish to claim that the lac operon cannot be considered as a model of gene regulation, only that the point of the PaJaMo experiment itself was not to provide knowledge that generalizes to physically distinct processes. The experiment was only supposed to generalize to other tokens of lac operon and repressor protein, even if the operon model can also be viewed as exemplifying a more general principle of gene regulation.
} 
So, my answer to the question of what distinguishes experimental models is the following: They are distinguished by being bona fide cases of scientific modeling that provide in vivo representations of some process. By an "in vivo representation" I mean such a representation that shows the typical features of a model, such as being idealized, simplified, multiply realizable, etc., but where the thing that does the representing is alive.

An important question is what it is that the model and its target have in common. I lack the space here to give this issue the treatment that it requires. To a first approximation, I suggest that what they have in common is some high-level functional properties such as being a predator. But this is an issue for further study.

\section{Why Model In Vivo Instead of In Silico?}

Why do biologists build experimental models? Can't they model and simulate the behavior of simple systems such as the ones examined here by using mathematical and computational tools? The answer is, of course, that they can and do so often. In fact, some of the work I have examined here also involved the use of such models along with the experimental model. So it seems that more ordinary forms of modeling go along with experimental modeling. So what is the added value of experimental modeling?

I suggest that the advantages differ from case to case. In example A, the experimental model offers avenues of research not available from mathematical models of evolutionary transitions simply for the lack of an appropriate theory. While there is a mathematically formulated theory to explain what the benefits of inter-cell cooperation are in evolutionary terms, there is no theory that would be able predict whether yeast or any other cells can evolve the cellular mechanisms of multicellurality within a few generations. Evolutionary theory can at best predict that multicellularity will evolve if the proximate mechanisms necessary for its realization are available. But that they can become available rapidly in an eukaryote had to be shown experimentally.

It's more difficult to say what the advantage of experimental modeling is in example B, because mathematical models that predict the behavior of two species engaged in a mutualistic interaction in ecological as well as evolutionary timescales are available. Here, there are two kinds of fact not predictable from evolutionary theory. The first kind is similar to example A: That the necessary mutations become available for evolution to act upon in both species is not predictable from evolutionary theory. This had to be shown empirically. The other kind of non-predictable facts concerns the occurrence of other changes that accompany the changes that the system was designed to manifest. In example B, the yeast strains engineered for obligatory mutualism (Shou et al. 2007) showed a tendency to increase their ability to survive population bottlenecks. That this would occur is, to my knowledge, not predictable from 
evolutionary theory. In the example of an engineered two-species trophic mutualism (Hillesland and Stahl 2010), the occurrence of antagonistic effects surprised the scientists. That this would occur was also not predictable from theory.

A helpful question to ask is what kind of surprises experimental models give rise to. Morgan (2003, 2005) distinguishes between results that "surprise" and results that "confound" the researcher. As I understand her distinction, a surprise is an unexpected behavior that is predictable from existing theory in principle and thus merely unexpected due to cognitive or computational limitations on the part of the modeler. Mathematical models, of course, can be full of surprises in this sense. By contrast, a result that confounds is "unexplainable within existing theory". It seems to me that the unexpected results in the experimental models are of the second kind. However, it should be noted that, unlike in the economics examples discussed by Morgan, this unexplainability is not due to any defect in the existing theory. It is simply not the job of evolutionary theory to explain what kinds of mutations are available to a species. This, I suggest, is an important reason why experimental modeling has been so successful in evolutionary biology.

Finally, it should be noted that important advantage of experimental models could be that the sources of potential error are different than in, for example, mathematical models. Unlike the latter, experimental models don't have to rely on the truth of certain theoretical assumptions. ${ }^{7}$ Instead, there are possible errors in the interpretation of data. Therefore, combining experimental with mathematical and computational modeling techniques, which was done in example B, could be an effective way of controlling for errors.

\section{Conclusions}

I hope to have established that there is a distinct kind of experimentation in biology that is best described as a form of scientific modeling. It consists of constructing model systems that are composed of living organisms (sometimes, but not necessarily genetically modified) and that are used as in vivo representations of biological processes in such a way that some processes are used as stand-ins for other processes. Unlike the classical model systems known from physics, such as the ideal gas, the model systems that experimental biologists construct are concrete and real, not abstract and not fictive. Furthermore, they are alive. But apart from that, they exhibit the characteristic features of scientific models, in particular idealization and surrogacy.

Experimental models, even though they are also experiments, differ from ordinary biological experiments mainly in the kinds of claims they are used to support: Their results are

\footnotetext{
${ }^{7}$ Thanks to Jon Williamson for pointing this out.
} 
Copyright Philosophy of Science 2014 Preprint (not copyedited or formatted)

Please use DOI when citing or quoting

generalized to other kinds of systems where the causal interactions under study are realized by physically distinct processes. 
Copyright Philosophy of Science 2014 Preprint (not copyedited or formatted)

Please use DOI when citing or quoting

\section{References}

Balagaddé, Frederick K., et al. 2008. "A Synthetic Escherichia coli Predator-prey Ecosystem." Molecular Systems Biology 4:187.

Burian, Richard M. 1997. "Exploratory Experimentation and the Role of Histochemical Techniques in the Work of Jean Brachet, 1938-1952." History and Philosophy of the Life Sciences 19:27-45.

Clarke, Ellen. 2012. "Plant Individuality: A Solution to the Demographer's Dilemma." Biology and Philosophy 27:321-361

Elliott, Kevin. 2007. "Varieties of Exploratory Experimentation in Nanotoxicology." History and Philosophy of the Life Sciences 29:311-334.

Frigg, Roman. 2010. "Models and Fiction." Synthese 172:251-268.

Frigg, Roman, and Stephan Hartmann. 2012. "Models in Science." In The Stanford Encyclopedia of Philosophy, ed. Edward N. Zalta. Stanford: The Metaphysics Research Lab, Center for the Study of Language and Information, Stanford University. $\mathrm{URL}=<\mathrm{http}: / /$ plato.stanford.edu/archives/fall2012/entries/models-science/ $>$

Giere, Ronald N. 2004. "How Models Are Used to Represent Reality." Philosophy of Science 71:742-752.

Griesemer, James R. 1990. "Material Models in Biology." In PSA 1990, Volume II, ed. Arthur Fine, Michael Forbes, and Linda Wessels, 79-93. East Lansing: Philosophy of Science Association.

Guala, Francesco. 2002. "Models, Simulations, and Experiments." In Model-based Reasoning: Science, Technology, Values, ed. Lorenzo Magnani and Nancy Nersessian, 59-74. Dordrecht: Kluwer.

Hillesland, Kristina L., and David A. Stahl. 2010. "Rapid Evolution of Stability and Productivity at the Origin of a Microbial Mutualism." Proceedings of the National Academy of Sciences U.S.A. 107:2124-2129.

Lenski, Richard E., and Michael Travisano. 1994. "Dynamics of Adaptation And Diversification: A 10,000-Generation Experiment With Bacterial Populations." Proceedings of the National Academy of Sciences of the United States of America 91:6808-6814.

Leonelli, Sabina, and Rachel Ankeny. 2011. "What's So Special About Model Organisms?" Studies in History and Philosophy of Science 42:313-323.

Levy, Arnon, and Adrian Currie. forthcoming. "Model Organisms Aren't (Theoretical) Models." The British Journal for the Philosophy of Science

Love, Alan C., and Michael Travisano. 2013. "Microbes Modeling Ontogeny." Biology and Philosophy 28:161-188.

Mäki, Uskali. 2005. "Models Are Experiments, Experiments Are Models." Journal of 
Copyright Philosophy of Science 2014 Preprint (not copyedited or formatted)

Please use DOI when citing or quoting

Economic Methodology 12:303-315

Morgan, M. S. 2003. "Experiments Without Material Intervention: Model Experiments, Virtual Experiments and Virtually Experiments." In The Philosophy of Scientific Experimentation, ed. Hans Radder, 216-235. Pittsburgh: The University of Pittsburgh Press,.

Morgan, Mary S. 2005. "Experiments versus Models: New Phenomena, Inference and Surprise." Journal of Economic Methodology 12: 317-329.

Morgan, Mary S., and Margaret Morrison, eds. 1999. Models as Mediators. Cambridge: Cambridge University Press.

O'Malley, Maureen. 2007. "Exploratory Experimentation and Scientific Practice:

Metagenomics and the Proteorhodopsin Case." History and Philosophy of the Life Sciences 29:335-358.

Park, Thomas. 1948. "Experimental Studies of Interspecies Competition. I. Competition Between Populations of the Flour Beetles, Tribolium confusum Duvall and Tribolium castaneum Herbst." Ecological Monographs 18:267-307.

Parker, Wendy S. 2009. "Does Matter Really Matter? Computer Simulations, Experiments, and Materiality." Synthese 169:483-496.

Plutynski, Anya. 2001. "Modeling Evolution in Theory and Practice." Philosophy of Science 68:S225-S236.

Ratcliff, William C., R. Ford Denison, Mark Borrello, and Michael Travisano. 2011. "Experimental Evolution of Multicellularity." Proceedings of the National Academy of Sciences U.S.A. 109:1595-1600

Rheinberger, Hans-Jörg. 1997. Toward a History of Epistemic Things: Synthesizing Proteins in the Test Tube. Stanford: Stanford University Press.

Shou, W., S. Ram, and J.M.G. Vilar. 2007. "Synthetic Cooperation in Engineered Yeast Populations." Proceedings of the National Academy of Sciences U.S.A. 104:18771882.

Timoféeff-Ressovsky, N.W. 1934. "Über den Einfluss des genotypischen Milieus und der Aussenbedingungen auf die Realisation des Genotyps." Nachrichten der Gesellschaft der Wissenschaften zu Göttingen, Biologie, N.F. 1:53-106.

Waters, C. Kenneth. 2012. "Experimental Modeling as a Form of Theoretical Modeling." Paper presented at the PSA 2012.

Weber, Marcel. 2005. Philosophy of Experimental Biology. Cambridge: Cambridge University Press.

Weber, Marcel. 2012. "Experiment in Biology." In The Stanford Encyclopedia of Philosophy, ed. Edward N. Zalta. Stanford: The Metaphysics Research Lab, Center for the Study of Language and Information, Stanford University. http://plato.stanford.edu/entries/biology-experiment/

Weisberg, Michael. 2007. "Who is a Modeler?" The British Journal for the Philosophy of Science 58:207-233. 
Copyright Philosophy of Science 2014 Preprint (not copyedited or formatted)

Please use DOI when citing or quoting

Weisberg, Michael. 2013. Simulation and Similarity: Using Models to Understand the World. Oxford: Oxford University Press.

Winsberg, Eric. 2009. "A Tale of Two Methods." Synthese 169:575-592. 